\title{
CCD sequences in the Shapley supercluster of galaxies. I. The LCO-Toronto telescope data ${ }^{\star, \star \star}$
}

\author{
L. Infante ${ }^{1}$, E. Slezak ${ }^{2}$ and H. Quintana ${ }^{\star \star \star}, 1$ \\ 1 Astronomy and Astrophysics Dept., P. Universidad Católica de Chile, Casilla 104, Santiago 22, Chile \\ 2 Observatoire de la Côte d'Azur, BP 229, 06304 Nice Cedex 4, France
}

Received March 18; accepted May 2, 1996

\begin{abstract}
We present $R$ CCD photometric sequences of stars for calibrating ESO/SERC (R) survey plates in the region of the Shapley supercluster of galaxies.
\end{abstract}

Key words: techniques: photometric — galaxies: photometry - surveys

\section{Introduction}

Recent analyses of automated catalogues of galaxies (Raychaudhury 1989) and of clusters of galaxies (Scaramella et al. 1989) made obvious the very rich concentration of galaxies in the Hydra-Centaurus region first noted by Shapley (1930). The intricated distribution of the galaxies in this region is related to the nearby HydraCentaurus supercluster of galaxies $\left(c z \simeq 4500 \mathrm{~km} \mathrm{~s}^{-1}\right)$ and to a more distant set of 14 Abell clusters lying within $10^{\circ}$ of A3558 ( $\left.c z \simeq 14000 \mathrm{~km} \mathrm{~s}^{-1}\right)$. This background high-density region is known as the Shapley supercluster. It appears to be one of the most massive structure in the local Universe and lies in the general direction of the dipole anisotropy of the cosmic microwave background. Since the late seventies our group is compiling photometric and spectroscopic data on the central clusters and neighbouring regions of the concentration in order to get masses for studying its overall dynamics (cf. Quintana et al. 1995). Velocity measurements in its inner part have also been used to get a more detailed view of the galaxy distribution inside this region (Bardelli et al. 1994) where large departures from the Hubble flow are likely to occur as well as interactions.

Despite these efforts, a better understanding is however still needed and we decided in 1992 to enlarge our study of the Shapley area by i) completing a spectroscopic survey

Send offprint requests to: E. Slezak

^Based on observations collected at the Las Campanas Observatory, Chile

${ }^{\star}$ Tables 1 to 15 are only published electronically and can be accessed by ftp at cdsarc.u-strasbg.fr or 130.79.128.5 or on www at http://cdsweb.u-strasbg.fr/abstract.html

${ }^{\star \star \star}$ Presidential Chair in Science of the galaxies brigther than $r_{\mathrm{F}}=17.5$ within $5^{\circ}$ of $\mathrm{A} 3558$ and of neighbouring clusters, and by ii) performing from the ESO/SERC $(R)$ photographic survey a $\sim 400$ square degrees automated galaxy count reaching a deeper magnitude limit than Raychaudhury's catalogue $\left(b_{j} \simeq 17\right)$. Both informations are necessary to get an accurate insight of the Shapley supercluster by addressing for instance questions like the connections in redshift space, the environmental effects on the formation and evolution of the structures, the dynamics of the supercluster, and the mapping of the mass distribution. Nevertheless, such a photometric catalogue will be quite useless if a reliable photometry is not provided with internal consistency over the involved ESO/SERC fields. Schmidt plates are known for exhibiting emulsion variations across their whole area and a monitoring of these variations is therefore required in order to get an accurate photometric calibration. In each field, many regularly distributed CCD frames have to be taken so that their pattern enables one to map the emulsion response while optimizing the observational time. We decided to obtain $13 \mathrm{CCD}$ sequences per plate : centre of the plate, centres of diagonals, and 3 spots along each edge. Such an extensive photometry is time consuming when applied to a large number of plates. Most of the Shapley supercluster region is hopefully covered by only 16 Schmidt fields and our accuracy aim is consequently within reach. Furthermore, as Cunow (1993) stated it within a similar context, we are only interested in good photometric zero points for a large number of fields rather than trying to achieve high photometric quality for individual objects.

We report here results of a first session of CCD photometry aimed to calibrate our scans of the ESO/SERC (R) survey plates achieved by the MAMA facility in Paris. Time limitations due to poor weather conditions during part of the 
run forced us to focus these observations on the central region of the supercluster and to limit to a few $(<5)$ the number of observed frames in the related Eso fields.

\section{CCD observations}

This first session of observations was carried out on February 17 to 22,1993 at the $0.6 \mathrm{~m}$ telescope of the University of Toronto at the Las Campanas Observatory in Chile. We used a back illuminated $516 \times 516$ pixel Thomson CCD, which provided us with a field of view of approximately $4^{\prime} \times 4^{\prime}$ and yielded frames with a $\sim 0.46^{\prime \prime}$ resolution. This telescope was manually operated including guiding, and special care was therefore needed to secure good image quality due to some mechanical instabilities in the tracking system. Although some CCD exposures had to be repeated due to this problem, the majority of them were suitable for doing photometry.

On the first two nights weather conditions were cloudy, but the remaining nights had photometric conditions under dark sky. Between 3 and 4 sequences taken from Landolt (1992) were observed in Johnson $V$ and $R$ bands at the beginning, middle and end of each night for $60 \mathrm{~s}$ to $180 \mathrm{~s}$ exposures. An average number of 35 standard stars per night was secured this way from sequences SA98, RUBIN149, PG0918+29, PG1323-086 and PG1633+099. The magnitude range of the measured stars is $11.2<R<14.8$ with a colour range of $-0.13<(V-R)<1.08$. To correct for pixel to pixel variations in sensitivity we observed the twilight sky in each filter to levels near 5000 counts/s every night.

The positions of the CCD fields were tuned in order to avoid bright SAO stars within the frames. The exposure time was set to $1200 s$ for each target field. The restricted amount of time with photometric conditions led to only 15 $R$ frames, 13 of which within the 3 ESO/SERC fields delineating the central region of the Shapley supercluster. The CCD frames which are pending to complete our Schmidt calibration program have been recorded during observational sessions held in 1994, 1995 and 1996. These later data will be described in forthcoming papers.

\section{Data reductions and photometry}

The CCD frames were treated using mainly the IRAF package (v. 2.10) for the image processing and aperture photometry tasks. After having computed the gain factor and the read-out noise of the CCD camera from raw bias and flat-field exposures, all frames have been first trimmed to remove some bad lines at the bottom and at the top. Next, they were corrected for the offsets due to the bias and the variations in pixel sensitivity. The bias which have been subtracted for each night was derived from the median averaging of individual bias frames where the median values of the columns (read-out axis) have been replicated in order to keep the noise level as low as possible. There was only a slight intensity difference between the bias exposures and the $1 \mathrm{~h}$ dark current ones, and a scaling with respect to the actual integration time was thus unnecessary. The flat-field frames taken in the same filter were rescaled to the same median value and pixel by pixel median averaged in order to eliminate possible star images. This yields to one median-averaged flat field in the instrumental $V$ band and another one in the $R$ band, which were used to get the data frames where aperture photometry has been carried out.

To process the standard frames, we used the PHOT task of IRAF which is designed for doing aperture photometry. About 5 to 10 standard stars had to be measured on each frame, so that we decided to run this program in an interactive way, both for the object selection and for the aperture settings. This way, the exact position of each star was determined from the implemented centroid algorithm applied within a pixel window around the flagged coordinates on the image. This window size was set individually between 4 and 8 pixels depending on the image seeing. In the same manner, the parameters of the annulus involved in the sky background evaluation were also interactively adjusted using the photometric profile of the objects. Each inner radius and width took into account close objects, and the mode of the histogram computed from the enclosed pixels was thereby a valuable estimate of the local sky background. Finally, the instrumental magnitudes were computed by integrating the sky-subtracted pixel values within an aperture the radius of which was also defined according to the object profile. This insured a correct aperture size and minimized therefore the magnitude error resulting from the sum over noisy background pixels. In order to transform these $\mathrm{CCD}$ magnitudes into the standard photometric system, we first introduced zeropoint $a_{0}$, airmass $a_{1}$ and colour $a_{2}$ corrections according to the following equation:

$$
R=R_{\mathrm{ccd}}+a_{0, R}+a_{1, R} X+a_{2, R}(V-R) .
$$

Using a least-mean square fitting program, the residual scatters for the 3 photometric nights of the run were then computed as equal to $\sigma^{(1)} \sim 0.06^{\mathrm{m}}, \sigma^{(2)} \sim 0.04^{\mathrm{m}}$ and $\sigma^{(3)} \sim 0.05^{\mathrm{m}}$ respectively. The colour terms were found to be pretty small : $a_{2}^{(1)} \simeq-0.066, a_{2}^{(2)} \simeq-0.022$, $a_{2}^{(3)} \simeq-0.031$. Since the Schmidt calibration exposures have only been obtained in the $R$ band, this result allowed us to remove the colour dependance from Eq. (1) without introducing a too large error. As a matter of fact, we finally decided to apply the restricted equation :

$$
R=R_{\mathrm{ccd}}+a_{0, R}^{\prime}+a_{1, R}^{\prime} X,
$$

and the residual scatters were indeed more or less the same as before: $\sigma^{\prime(1)} \sim 0.07^{\mathrm{m}}, \sigma^{\prime(2)} \sim 0.04^{\mathrm{m}}$ and $\sigma^{\prime(3)} \sim 0.05^{\mathrm{m}}$. The set of calibration exposures contains a large number of objects and had to be processed automatically. These 
objects would have to be detected in a batch mode using the DAOFIND task of IRAF. However, such lists of coordinates are quite sensitive to some required parameter definitions, namely to the ellipticity and the full width at half maximum of the Gaussian function which is used to look for brightness enhancements in convolved data, and to the morphological characteristics of the searched astronomical objects. Even if we were not interested in getting a catalogue complete down to the detection limit, a complete enough count would have required a fine tuning of these parameters for each image in order to get rid of the peculiar guiding errors they are suffering from. So, we decided to detect and to measure the objects in the calibration frames using our own software which involves a thresholding at a given level above the local sky background. This background was obtained from the mode of the pixel intensity distribution in boxes of $128 \times 128$ pixels followed by a bilinear interpolation of these values, while the detection threshold was set to two rms deviations. Then, a unique size for the circular aperture photometry was chosen with respect to the brightest objects to be correctly measured, which led to a radius of 20 to 30 pixels depending on the processed frame. Defining a set of radii was indeed quite useless due to the geometrical deformations cited above. The actual background values subtracted for the photometric computations were obtained from the mode of the pixel intensities within a 10 pixel width annulus contiguous to the aperture. All the sky-subtracted pixels within this sole aperture were finally added to get the instrumental magnitude for the detected objects, the positions of which result from the barycentres of the thresholded adjacent pixels. The photometric errors were estimated on some isolated stars using the facility included in the PHOT task with the same aperture as in our code. These random errors are computed from the local $\mathrm{s} / \mathrm{N}$ ratio. They were found to be less than $0.002^{\mathrm{m}}$ for stars brighter than $R=14$, and to be equal to about $0.003^{\mathrm{m}}, 0.007^{\mathrm{m}}, 0.012^{\mathrm{m}}, 0.019^{\mathrm{m}}$ and $0.030^{\mathrm{m}}$ for stars with $14<R<15,15<R<16,16<R<17,17<R<18$, and $18<R<19$ respectively. These values are similar to the differences in magnitude for the very few objects measured twice on different frames (cf. objects 5 and 6 in Tables 9 and 10).

\section{Results}

Since the pixel data from the MAMA scans will be linearized according to the internal calibration procedure described by Bunclark \& Irwin (1983), the final catalogue of each program field has been designed to include only isolated stars with a $\mathrm{S} / \mathrm{N}$ ratio high enough. Our goal was of course to secure the future Schmidt zero-points not only from a statistical point of view but also from the intrinsic quality of the involved measurements. To do so, close objects were first removed when overlapped measurements could have occurred. The aperture size was indeed chosen quite large to take into account the systematic elongation due to the unperfect guiding system. Then, the stars were picked out by selecting them from their distinctive brightness with respect to diffuse extended objects, a check being done by a visual inspection of their isophotal contours on the Schmidt scans. The efficiency of this procedure is unlikely to be high for objects fainter than $R=19$ and thus with a low S/N. Such objects have therefore been eliminated from the final list. Finally, a test rejected all the objects which misbehaved according to a plot of the CCD total magnitudes against the scans on-line flux estimates (composite or peculiar objects, erroneous sky background value, etc.).

The CCD sequences are given in Tables 1 to 15. The equatorial coordinates of each objet are those of its counterpart in the catalogue obtained from the ESO/SERC (R) scans. Actually, the inspection of the CCD frames allowed an easy identification of the few bright selected objects in the covered areas with the MAMA, which provided object lists in the corresponding regions. So, the positional accuracy of the astrometric reduction is about $0.5^{\prime \prime}$.

Acknowledgements. We are thankful to Dr. R. Garrison and to the Time Committee of the Toronto telescope for allocation of telescope time. We would also like to thank M. Way for carrying out part of the observations. We gratefully acknowledge partial support from the France-Chile cooperation program C93U02 under the ECOS (Paris) and CONICYT (Santiago) committes, and funding from the FONDECYT grants 1930570 (to L. Infante) and 1930572 (to H. Quintana). H. Quintana gratefully acknowledges the award of a Presidential Chair in Science.

\section{References}

Bardelli S., Zucca E., Vettolani G., Zamorani G., Scaramella R., et al., 1994, MNRAS 267, 665

Bunclark P.S., Irwin M.J., 1983, in Symp. on Statistical methods in Astronomy, ESA SP-201, p. 195

Cunow B., 1993, A\&AS 97, 541

Landolt A.U., 1992, AJ 104, 340

Quintana H., Ramirez A., Melnick J., Raychaudhury S., Slezak E., 1995, AJ 110, 463

Raychaudhury S., 1989, Nat 342, 251

Scaramella R., Baiesi-Pillastrini G., Chincarini G., Vettolani G, 1989, Nat 338, 562

Shapley H., 1930, Harvard Coll. Obs. Bull. 874, 9 